\title{
Mulliken Population Analysis of X-Ray Magnetic Circular Dichroism in Uranium Monochalcogenides: Examination of Sum Rules by Fully Relativistic Full-Potential LCAO Method
}

\author{
Shugo Suzuki, Toshihiro AriIzumi, and Ming-Fang Li \\ Institute of Materials Science, University of Tsukuba, Tsukuba 305-8573
}

(Received May 25, 2009)

\begin{abstract}
We study the X-ray magnetic circular dichroism (XMCD) spectra at the $\mathrm{U} M_{4,5}$ and $N_{4,5}$ edges of uranium monochalcogenides, $\mathrm{U} X$ where $X=\mathrm{S}$, Se, and $\mathrm{Te}$, examining the applicability of the XMCD sum rules to $U X$ by the fully relativistic full-potential linearcombination-of-atomic-orbitals method based on the density functional theory. To extract the transitions relevant to the sum-rule analysis, we employ the Mulliken population analysis (MPA). Using the MPA, the orbital sum rule is found to be valid to $10-20 \%$ for the $M_{4,5}$ edges and valid to $5-15 \%$ for the $N_{4,5}$ edges. On the other hand, the spin sum rule is found to be valid to $10-20 \%$ for the $M_{4,5}$ edges whereas valid to $30-35 \%$ for the $N_{4,5}$ edges. Furthermore, it is found that the calculated XMCD spectra are consistent with a recent experimental observation that the intensity of the $N_{4,5} \mathrm{XMCD}$ signal is comparable to that of the $M_{4,5}$ XMCD signal although contradicting a previous theoretical prediction that the XMCD intensity at the $N_{4,5}$ edges is one order of magnitude smaller than that at the $M_{4,5}$ edges.
\end{abstract}

KEYWORDS: Mulliken population analysis, X-ray magnetic circular dichroism, uranium monochalcogenides, sum rules, fully relativistic full-potential LCAO method

\section{Introduction}

Since the observation of the X-ray magnetic circular dichroism (XMCD), ${ }^{1)}$ the magnetism of the ferromagnetic materials with transition, rare-earth, and actinide elements has been investigated extensively analyzing their XMCD spectra together with the X-ray absorption spectroscopy (XAS) spectra. ${ }^{2-31)}$ Important experimental and theoretical works have been done so far, allowing us to study the magnetism from different specific atoms using the high element selectivity of the XMCD technique. In particular, the XMCD sum rules have been found to be a powerful tool for determining the orbital and spin contributions to the magnetization separately. ${ }^{14-17)}$

The applicability of the XMCD sum rules to $3 d$ transition metals was examined theoretically from first principles using the full-potential linearized augmented plane wave (FLAPW) method based on the density functional theory; ${ }^{18-20)}$ the XMCD sum rules are derived from a single ion model, and hence it is important to verify their applicability to real materials such as $3 d$ transition metals, which have strongly hybridized multiband structures. In study- 
ing the XMCD theoretically, it is crucial to deal with spin-orbit coupling (SOC) accurately because the XMCD is originated in SOC. For this purpose, the FLAPW study adopted the second variation procedure where SOC is considered as a small perturbation. As a result, it was shown that the orbital sum rule is valid to $5-10 \%$ whereas the spin sum rule is valid to about $50 \%$.

On the other hand, there have been no first-principles studies that examine the applicability of the XMCD sum rules to the ferromagnetic materials with actinide elements although several theoretical studies of the XMCD in this class of material have been done. ${ }^{21-28)}$ Typical materials among them are uranium monochalcogenides, $\mathrm{U} X$ where $X=\mathrm{S}$, Se, and Te. The most important difference between $3 d$ transition metals and $\mathrm{U} X$ is the magnitude of SOC; SOC in the latter materials is so large that the orbital contribution to the magnetization are sizable. For this reason, in studying UX theoretically, fully relativistic calculations are indispensable without recourse to a perturbative approach. ${ }^{32)}$ Furthermore, since there have already been several experimental studies of the XMCD in UX employing the sum-rule analysis, ${ }^{29-31)}$ the applicability of the XMCD sum rules to UX should be examined theoretically.

When using the XMCD sum rules, of great importance is to extract the transitions to be considered in the sum-rule analysis because there exist extra contributions of other transitions to the measured or calculated spectra. This has been emphasized in the previous FLAPW study, in which a proper energy cutoff for the integration was introduced in order to eliminate the error originated in the high lying energy states. ${ }^{18-20)}$ The reason why the extraction procedure is needed is that the XMCD sum rules are derived assuming that the transitions are from a single core shell to a single valence shell, with both shells being specified by their respective principal and azimuthal quantum numbers. Instead of the use of the energy cutoff, one can also use the Mulliken population analysis (MPA) in order to extract the transitions relevant to the sum-rule analysis. The MPA is a standard procedure widely used in the linearcombination-of-atomic-orbitals (LCAO) method for decomposing a physical quantity into the atomic constituent parts comprising the quantity as a whole. ${ }^{33)}$

In this study, we investigate the XMCD at the $\mathrm{U} M_{4,5}$ and $N_{4,5}$ edges of $\mathrm{U} X$ and examine the applicability of the XMCD sum rules to $\mathrm{UX}$, using the fully relativistic full-potential LCAO (FFLCAO) method based on the density functional theory within the local spin density approximation (LSDA). Here, the MPA is employed as a procedure for extracting the transitions relevant to the sum-rule analysis. We also compare our results with experimental data available. In particular, we study the magnitude of the XMCD signal to resolve the contradiction between a recent experimental observation and a previous theoretical prediction; the recent experimental study concluded that the intensity of the $N_{4,5}$ XMCD signal is comparable to that of the $M_{4,5}$ XMCD signal whereas the previous theoretical study predicted that the XMCD intensity at the $N_{4,5}$ edges is one order of magnitude smaller than that at the 
$M_{4,5}$ edges. In $\S 2$, we describe the method of calculations, explaining the MPA of the optical conductivity tensor in detail. The results and discussion are given in $\S 3$. Finally, we give the conclusions of this study in $\S 4$.

\section{Method of Calculations}

The XAS and XMCD spectra are calculated using the FFLCAO method as follows. ${ }^{34-36)}$ Firstly, we calculate the optical conductivity tensor $\sigma_{\xi \eta}$ as a function of photon energy $\hbar \omega$. In calculating $\sigma_{\xi \eta}$, we use the fully relativistic expression within the electric-dipole approximation. ${ }^{35)}$ The expression involves the matrix elements of the Dirac matrices $\boldsymbol{\alpha}$ as the transition matrix elements between the initial state $|l \boldsymbol{k}\rangle$ and the final state $|n \boldsymbol{k}\rangle$ :

$$
\begin{aligned}
\sigma_{\xi \eta}= & \frac{2 i e^{2} c^{2}}{\hbar \Omega} \sum_{\boldsymbol{k}} \sum_{l} \sum_{n} \frac{f_{l \boldsymbol{k}}\left(1-f_{n \boldsymbol{k}}\right)}{\omega_{\tau}^{2}-\omega_{n \boldsymbol{k}}^{2}} \\
& \times\left\{\frac{\omega_{\tau}}{\omega_{n l \boldsymbol{k}}} \operatorname{Re}\left[\left\langle l \boldsymbol{k}\left|\alpha_{\xi}\right| n \boldsymbol{k}\right\rangle\left\langle n \boldsymbol{k}\left|\alpha_{\eta}\right| l \boldsymbol{k}\right\rangle\right]+i \operatorname{Im}\left[\left\langle l \boldsymbol{k}\left|\alpha_{\xi}\right| n \boldsymbol{k}\right\rangle\left\langle n \boldsymbol{k}\left|\alpha_{\eta}\right| l \boldsymbol{k}\right\rangle\right]\right\} .
\end{aligned}
$$

Here, $\boldsymbol{k}$ denotes the wave number and $\omega_{n l \boldsymbol{k}}$ the energy difference between the initial and final states, $\left(\varepsilon_{n \boldsymbol{k}}-\varepsilon_{l \boldsymbol{k}}\right) / \hbar$. Also, $\omega_{\tau}$ denotes $\omega+i / \tau$ with $\tau$ being the lifetime of excited electrons, $\Omega$ the volume of the unit cell, and $f_{l \boldsymbol{k}}$ the occupation number of $|l \boldsymbol{k}\rangle$. Moreover, we consider only the interband contribution to the optical conductivity tensor because the XAS and XMCD spectra are not affected by the intraband contribution. Next, using the optical conductivity tensor, we calculate the real part $n_{ \pm}$and the imaginary part $\kappa_{ \pm}$of the complex refractive index; the plus and minus signs refer to the left and right circular polarizations, respectively. The complex refractive index is given as

$$
n_{ \pm}+i \kappa_{ \pm}=\sqrt{\varepsilon_{x x} \pm i \varepsilon_{x y}},
$$

where $\varepsilon_{\xi \eta}$ is the dielectric tensor defined as $\varepsilon_{\xi \eta}=\delta_{\xi \eta}+4 \pi i \sigma_{\xi \eta} / \omega$. Finally, we calculate the $\mathrm{XAS}$ and XMCD spectra using the imaginary part of the complex refractive index $\kappa_{ \pm}$. The $\mathrm{XAS}$ and XMCD spectra are given as

$$
\mu_{\mathrm{tot}}=\frac{2 \omega}{c}\left(\kappa_{+}+\kappa_{-}\right)
$$

and

$$
\Delta \mu=\frac{2 \omega}{c}\left(\kappa_{+}-\kappa_{-}\right),
$$

respectively. In addition, the absorption coefficient for light linearly polarized along the $z$ axis,

$$
\mu_{z}=\frac{2 \omega}{c} \kappa_{z}
$$

where $\kappa_{z}=\operatorname{Im} \sqrt{\varepsilon_{z z}}$, is used in the sum-rule analysis. ${ }^{14,15)}$

In this study, we analyze the XAS and XMCD spectra decomposing them into the contributions of individual transitions. For this purpose, we adopt the MPA. ${ }^{33)}$ Firstly, the one-electron 
state $|l \boldsymbol{k}\rangle$ is expanded as

$$
|l \boldsymbol{k}\rangle=\sum_{p} C_{p l}^{\boldsymbol{k}}|p \boldsymbol{k}\rangle
$$

where $|p \boldsymbol{k}\rangle$ is the Bloch sum of the $p$ th atomic orbitals:

$$
|p \boldsymbol{k}\rangle=\sum_{u}\left|\chi_{p u}\right\rangle \exp \left(i \boldsymbol{k} \cdot \boldsymbol{R}_{u}\right)
$$

with $\left|\chi_{p u}\right\rangle$ being the $p$ th atomic orbital in the $u$ th unit cell whose translation vector is $\boldsymbol{R}_{u}$. We next consider the normalization condition of $|l \boldsymbol{k}\rangle$ :

$$
\langle l \boldsymbol{k} \mid l \boldsymbol{k}\rangle=\sum_{p} \sum_{q} C_{p l}^{\boldsymbol{k} *} S_{p q}^{\boldsymbol{k}} C_{q l}^{\boldsymbol{k}}=1
$$

with $S_{p q}^{\boldsymbol{k}}$ being the overlap integral $\langle p \boldsymbol{k} \mid q \boldsymbol{k}\rangle$. The Mulliken population of the $p$ th atomic orbital in the one-electron state $|l \boldsymbol{k}\rangle$ is then defined as

$$
Q_{p}^{l \boldsymbol{k}}=\operatorname{Re}\left(\sum_{q} C_{p l}^{\boldsymbol{k} *} S_{p q}^{\boldsymbol{k}} C_{q l}^{\boldsymbol{k}}\right) .
$$

This represents the fraction of the $p$ th atomic orbital in the one-electron state $|l \boldsymbol{k}\rangle$. The normalization condition of the one-electron state $|l \boldsymbol{k}\rangle$ is now rewritten as

$$
\sum_{p} Q_{p}^{l k}=1
$$

Using $Q_{p}^{l k}$, we decompose the optical conductivity tensor as follows:

$$
\sigma_{\xi \eta}=\sum_{p} \sum_{q} \sigma_{\xi \eta}^{p q}
$$

where

$$
\begin{aligned}
\sigma_{\xi \eta}^{p q}= & \frac{2 i e^{2} c^{2}}{\hbar \Omega} \sum_{\boldsymbol{k}} \sum_{l} \sum_{n} Q_{p}^{l \boldsymbol{k}} Q_{q}^{n \boldsymbol{k}} \frac{f_{l \boldsymbol{k}}\left(1-f_{n \boldsymbol{k}}\right)}{\omega_{\tau}^{2}-\omega_{n l \boldsymbol{k}}^{2}} \\
& \times\left\{\frac{\omega_{\tau}}{\omega_{n l \boldsymbol{k}}} \operatorname{Re}\left[\left\langle l \boldsymbol{k}\left|\alpha_{\xi}\right| n \boldsymbol{k}\right\rangle\left\langle n \boldsymbol{k}\left|\alpha_{\eta}\right| l \boldsymbol{k}\right\rangle\right]+i \operatorname{Im}\left[\left\langle l \boldsymbol{k}\left|\alpha_{\xi}\right| n \boldsymbol{k}\right\rangle\left\langle n \boldsymbol{k}\left|\alpha_{\eta}\right| l \boldsymbol{k}\right\rangle\right]\right\} .
\end{aligned}
$$

The above decomposition is a straightforward extension of the decomposition of the total density states into the partial densities of states, as widely used in the LCAO calculations. ${ }^{36}$ ) In the X-ray region, since the order of the magnitude of $\sigma_{\xi \eta} / \omega$ is less than $10^{-3}$, the following approximation is satisfactory:

$$
n_{ \pm}+i \kappa_{ \pm} \simeq 1+2 \pi i\left(\sigma_{x x} \pm i \sigma_{x y}\right) / \omega .
$$

As a result, we have

$$
\mu_{\mathrm{tot}}=\frac{8 \pi}{c} \operatorname{Re} \sigma_{x x}
$$

and

$$
\Delta \mu=-\frac{8 \pi}{c} \operatorname{Im} \sigma_{x y}
$$


That is, in the X-ray region, the decomposition of the XAS and XMCD spectra into the contributions of individual transitions is meaningful as in the MPA of the optical conductivity tensor.

We calculate the orbital and spin angular momenta using the XMCD sum rules as follows. ${ }^{14,15)}$ Firstly, the orbital sum rule is given as

$$
\rho=\frac{\left\langle L_{z}\right\rangle}{3\left(14-n_{5 f}\right)}=\frac{\int_{E_{4}+E_{5}} \Delta \mu \mathrm{d} \omega}{\int_{E_{4}+E_{5}}\left(\mu_{\mathrm{tot}}+\mu_{z}\right) \mathrm{d} \omega},
$$

where $\left\langle L_{z}\right\rangle$ is the expectation value of the $z$ component of the orbital angular momentum operator. Secondly, the spin sum rule is given as

$$
\delta=\frac{2\left\langle S_{\mathrm{e}}\right\rangle}{3\left(14-n_{5 f}\right)}=\frac{\int_{E_{5}} \Delta \mu \mathrm{d} \omega-\frac{3}{2} \int_{E_{4}} \Delta \mu \mathrm{d} \omega}{\int_{E_{4}+E_{5}}\left(\mu_{\mathrm{tot}}+\mu_{z}\right) \mathrm{d} \omega},
$$

where $\left\langle S_{\mathrm{e}}\right\rangle$ is the effective spin angular momentum:

$$
\left\langle S_{\mathrm{e}}\right\rangle=\left\langle S_{z}\right\rangle+3\left\langle T_{z}\right\rangle
$$

with $\left\langle S_{z}\right\rangle$ and $\left\langle T_{z}\right\rangle$ being the expectation values of the $z$ components of the spin angular momentum operator and the magnetic dipole operator, respectively; the magnetic dipole operator is defined as $T_{z}=\sum_{i}\left[s_{z i}-3 z_{i}\left(\boldsymbol{r}_{i} \cdot \boldsymbol{s}_{i}\right) / r_{i}^{2}\right]$ with $\boldsymbol{s}_{i}$ and $\boldsymbol{r}_{i}$ being the spin operator and position vector of the $i$ th electron, respectively. In the above expressions, $E_{4}\left(E_{5}\right)$ represents $M_{4}\left(M_{5}\right)$ for the $M_{4,5}$ edges and $N_{4}\left(N_{5}\right)$ for the $N_{4,5}$ edges, respectively, and $n_{5 f}$ represents the number of electrons in the $\mathrm{U} 5 f$ shell. It should be noted that, in addition to the XAS and XMCD spectra, we need $n_{5 f}$ for determining $\left\langle L_{z}\right\rangle$ and $\left\langle S_{\mathrm{e}}\right\rangle$ although we do not need it for determining $\rho$ and $\delta$.

The FFLCAO calculations were carried out as follows. The experimental lattice constants of US, USe, and UTe, which crystallize in the $\mathrm{NaCl}$ structure exhibiting a strong magnetic anisotropy with an easy axis in the [111] direction, are 5.489, 5.740, and 6.155 $\AA$, respectively, ${ }^{37)}$ and we used these experimental values. We assumed that both the magnetization axis and the direction of incident X-ray are in the [111] direction, which was taken as the $z$ axis in our calculations. Also, we used the following four-component atomic orbitals: $1 s, 2 s$, $2 p, 3 s, 3 p, 3 d, 4 s, 4 p, 4 d, 4 f, 5 s, 5 p, 5 d, 5 f, 6 s, 6 p, 6 d$, and $7 s$ orbitals of the neutral $\mathrm{U}$ atom, $5 f, 7 s$, and $7 p$ orbitals of the $\mathrm{U}^{2+}$ atom, $1 s, 2 s, 2 p, 3 s$, and $3 p$ atomic orbitals of the neutral $\mathrm{S}$ atom, and $3 s, 3 p$, and $3 d$ orbitals of the $\mathrm{S}^{2+}$ atom, $1 s, 2 s, 2 p, 3 s, 3 p, 3 d, 4 s$, and $4 p$ atomic orbitals of the neutral Se atom, and $4 s, 4 p$, and $4 d$ orbitals of the $\mathrm{Se}^{2+}$ atom, $1 s, 2 s, 2 p$, $3 s, 3 p, 3 d, 4 s, 4 p, 4 d, 5 s$, and $5 p$ atomic orbitals of the neutral Te atom, and $5 s, 5 p$, and $5 d$ orbitals of the $\mathrm{Te}^{2+}$ atom. It is necessary to use not only the atomic orbitals of neutral atoms but also those of positively charged atoms for describing the contraction of atomic orbitals associated with cohesion. Furthermore, we carried out real-space integration using 4644 points for the U atom, 2580 points for the $\mathrm{S}$ atom, 3096 points for the Se atom, and 3612 points 
for the Te atom. Also, we used the LSDA exchange-correlation potential represented by the Perdew-Zunger parameterization of Ceperly-Alder results. ${ }^{38,39)}$ The Brillouin-zone integration was carried out using the good-lattice-point method with $185 \boldsymbol{k}$ points. ${ }^{40)}$ These conditions of calculations were confirmed previously to be sufficient for studying the electronic properties as well as the magnetic ones. ${ }^{36)}$ Also, in calculating the optical conductivity tensor, we used the lifetime parameter $\hbar / \tau=2.5 \mathrm{eV}$ throughout.

\section{Results and discussion}

\section{$3.1 X A S$ and $X M C D$ at $M_{4,5}$ edges}

We show the calculated XAS and XMCD spectra at the U $M_{4,5}$ edges of US, USe, and UTe in Figs. 1, 2, and 3, respectively. In these figures, (a) the XAS spectra, (b) the XMCD spectra at the $M_{5}$ edge, and (c) the XMCD spectra at the $M_{4}$ edge are shown. Solid lines represent the total spectra involving all transitions. On the other hand, dashed lines represent the spectra originated in the $\mathrm{U} 3 d \rightarrow 5 f$ transitions extracted using the MPA.

We begin with the XAS spectra. The spectra of US, USe, and UTe are similar to each other. The XAS spectra consist of the main $M_{5}$ and $M_{4}$ peaks and the structures at the high-energy sides of the main peaks. It is found that the $\mathrm{U} 3 d \rightarrow 5 f$ transitions contribute dominantly to the main peaks. Using the MPA, we found that the high-energy structures originate mainly in the $\mathrm{U} 3 d \rightarrow 6 f$ transitions. It should however be noted that the $\mathrm{U} 6 f$ states or, more precisely, the $\mathrm{U} 6 f$-like states are no longer atomic states because these states are delocalized considerably with significant hybridization with chalcogen states. For all the compounds, the calculated energy position of the $M_{5}$ peak is $3.49 \mathrm{keV}$ and that of the $M_{4}$ peak is $3.67 \mathrm{keV}$. The experimental energy position of the $M_{5}$ peak is $3.55 \mathrm{keV}$ and that of the $M_{4}$ peak is $3.73 \mathrm{keV} .{ }^{30}$ ) Thus, the calculated XAS spectra are found to shift to lower energy by about $0.06 \mathrm{keV}$; the calculation error is about $2 \%$. Also, the calculated and experimental values of spin-orbit splitting, both being $0.18 \mathrm{keV}$, are in excellent agreement with each other. In the experimental XAS spectra of US, there exists a very broad peak at $0.03 \mathrm{keV}$ from each main peak. ${ }^{29,30)}$ These broad peaks may correspond to our calculated high-energy structures. However, the experimental high-energy structures are considerably broader than the calculated ones. This might be caused by the insufficiency of the basis set used in our calculations; the basis functions of shorter wave length should be added for describing the electronic states in the high-energy region more appropriately. The total integrated intensity decreases from US to UTe. The ratio of the total integrated intensities of USe and UTe to that of US is 0.86 and 0.67 , respectively, if we consider the total spectra. On the other hand, if we consider only the U $3 d \rightarrow 5 f$ spectra, the ratio of USe and UTe to US is 0.86 and 0.66 , respectively. The difference between the values of the ratio calculated considering the total spectra and those calculated considering only the $\mathrm{U} 3 d \rightarrow 5 f$ spectra is not very large. Next, we examine the branching ratio, which is defined as $A_{5 / 2} /\left(A_{5 / 2}+A_{3 / 2}\right)$ with $A_{5 / 2}$ and $A_{3 / 2}$ being the 
integrated intensities of the $M_{5}$ and $M_{4}$ XAS peaks, respectively. The calculated branching ratio is found to be $0.63,0.64$, and 0.65 for UT, USe, and UTe, respectively, if we consider the total spectra. On the other hand, if we consider only the $\mathrm{U} 3 d \rightarrow 5 f$ spectra, the calculated branching ratio is found to be $0.65,0.66$, and 0.67 for US, USe, and UTe, respectively. The difference between the values of the branching ratio calculated considering the total spectra and those calculated considering only the $\mathrm{U} 3 d \rightarrow 5 f$ spectra is not very large. Also, the branching ratio increases slightly from US to UTe.

We next examine the XMCD spectra. The spectra of US, USe, and UTe are similar to each other. The $M_{5}$ spectra exhibit asymmetric lineshape while the $M_{4}$ spectra exhibit symmetric line shape. Furthermore, the integrated intensities of the $M_{5}$ and $M_{4}$ spectra have the same sign. This means that there exists a large orbital angular momentum as shown later by the sum-rule analysis. It should however be noted that the integrated intensities of the $M_{4}$ spectra are much larger than those of the $M_{5}$ spectra. The ratio of the integrated intensities of the $M_{5}$ spectra to those of the $M_{4}$ spectra is $0.19,0.20$, and 0.22 for US, USe, and UTe, respectively, if we consider the total spectra. On the other hand, if we consider only the $\mathrm{U} 3 d \rightarrow 5 f$ spectra, the $M_{5}$ to $M_{4}$ ratio is $0.26,0.30$, and 0.36 for US, USe, and UTe, respectively. It is also found that the ratio increases slightly from US to UTe. The $M_{5}$ to $M_{4}$ ratio experimentally observed in US is $0.13 \pm 0.03 .^{29)}$ Thus, the calculated $M_{5}$ to $M_{4}$ ratio of US, 0.26 , is larger than the experimental one. The total integrated intensity decreases from US to UTe. The ratio of the total integrated intensities of USe and UTe to that of US is 0.98 and 0.93 , respectively, if we consider the total spectra. On the other hand, if we consider only the $\mathrm{U} 3 d \rightarrow 5 f$ spectra, the ratio of USe and UTe to US is 0.99 and 0.96 , respectively. The difference between the values of the ratio calculated considering the total spectra and those calculated considering only the $\mathrm{U} 3 d \rightarrow 5 f$ spectra is not very large.

It was mentioned above that our calculated $M_{5}$ to $M_{4}$ ratio is larger than the experimental one. We here discuss a possible origin of the overestimation of the $M_{5}$ to $M_{4}$ ratio. The previous Hartree-Fock study of US has shown that the $M_{5}$ to $M_{4}$ ratio depends strongly on the treatment of the spin-off-diagonal operators. ${ }^{22)}$ One important point is to express the oneelectron wavefunctions as a linear combination of spin-up and spin-down states appropriately because SOC mixes the spin-up and spin-down states. Another important point is to consider the spin-off-diagonal terms in the exchange potential. In our calculations, although the former point is taken into account, the latter point is not because the LSDA exchange potential adopted in this study takes account of only the spin-diagonal terms. Actually, the HartreeFock study has shown that the neglect of the spin-off-diagonal terms in the exchange potential results in the overestimation of the $M_{5}$ to $M_{4}$ ratio. It is most likely that the overestimation of our calculated $M_{5}$ to $M_{4}$ ratio are due to the neglect of the spin-off-diagonal terms in the LSDA exchange potential. It will be found later in the sum-rule analysis that our calculated 
$\left\langle T_{z}\right\rangle$ is smaller than expected; this may be due to the same reason, considering the results of the Hartree-Fock study.

\subsection{XAS and XMCD at $N_{4,5}$ edges}

We now show the calculated XAS and XMCD spectra at the U $N_{4,5}$ edges of US, USe, and UTe in Figs. 4, 5, and 6, respectively. In these figure, (a) the XAS spectra, (b) the XMCD spectra at the $N_{5}$ edge, and (c) the XMCD spectra at the $N_{4}$ edge are shown. Solid lines represent the total spectra involving all transitions. On the other hand, dashed lines represent the spectra originated in the $\mathrm{U} 4 d \rightarrow 5 f$ transitions extracted using the MPA.

The XAS spectra consist of the main $N_{5}$ and $N_{4}$ peaks and the structures at the highenergy sides of the main peaks. It is found that the $\mathrm{U} 4 d \rightarrow 5 f$ transitions contribute dominantly to the main peaks. Using the MPA, we found that the high-energy structures originate mainly in the $\mathrm{U} 4 d \rightarrow 6 f$ transitions; it should again be noted that " $6 f$ " means " $6 f$-like" as already mentioned in the above. For all the compounds, the calculated energy position of the $N_{5}$ peak is $706 \mathrm{eV}$ and that of the $N_{4}$ peak is $747 \mathrm{eV}$. The experimental energy position of the $N_{5}$ peak is $737 \mathrm{eV}$ and that of the $N_{4}$ peak is $778 \mathrm{eV}{ }^{31)}$ Thus, the calculated XAS spectra are found to shift to lower energy by about $31 \mathrm{eV}$; the calculation error is about 4 \%. Also, the calculated and experimental spin-orbit splittings, both being $41 \mathrm{eV}$, are again in excellent agreement with each other. In the experimental XAS spectra of US and USe, it is difficult to find the peaks corresponding to the calculated high-energy structures. ${ }^{31)}$ However, the line shape analysis has clearly shown that there exists a background contribution at the high-energy side of each main peak. These background contributions may correspond to our calculated high-energy structures. However, to reproduce the background contribution appropriately, the basis functions of shorter wave length should be added to the basis set as noted in the previous subsection. One of the differences between the XAS spectra at the U $M_{4,5}$ edges and those at the $\mathrm{U} N_{4,5}$ edges is the energy region of the spectra; the calculated $\mathrm{U} N_{4,5}$ peaks are in the soft X-ray region while the calculated $\mathrm{U} M_{4,5}$ peaks are in the hard X-ray region. Another difference is the magnitude of spin-orbit splitting; the calculated spin-orbit splitting at the $\mathrm{U} N_{4,5}$ edges is $41 \mathrm{eV}$ while that at the $\mathrm{U} M_{4,5}$ edges is $180 \mathrm{eV}$. Next, we analyze the ratio of the total integrated intensities of USe and UTe to that of US. The ratio of the total integrated intensities of USe and UTe to that of US is 0.86 and 0.67 , respectively, if we consider the total spectra. On the other hand, if we consider only the $\mathrm{U} 4 d \rightarrow 5 f$ spectra, the ratio of USe and UTe to US is 0.86 and 0.66 , respectively. Thus, the total integrated intensity is found to decrease from US to UTe by the same fraction as in the XAS spectra at the U $M_{4,5}$ edges, if we consider only the $4 d \rightarrow 5 f$ spectra. We now analyze the branching ratio. The analysis is however found to be difficult if we consider the total spectra because there is a substantial overlap between the $N_{5}$ and $N_{4}$ intensities. For this reason, we analyze the branching ratio only for the $\mathrm{U} 4 d \rightarrow 5 f$ spectra. The calculated branching ratio is then found 
to be $0.68,0.69$, and 0.70 for US, USe, and UTe, respectively. The branching ratio increases slightly from US to UTe. The branching ratio is also almost the same as those in the $M_{4,5}$ spectra although there exists a small difference of about $5 \%$. For the $N_{4,5}$ spectra of US and USe, there are experimentally determined values of the branching ratio available, which were obtained using the line shape analysis; ${ }^{31)}$ the branching ratio for US is 0.70 and that for USe is 0.73 . Our calculated results, 0.68 and 0.69 , are in reasonable agreement with theses experimental values, both increasing slightly from US to USe, although the agreement is not perfect.

We next examine the XMCD spectra at the U $N_{4,5}$ edges. The spectra of US, USe, and UTe are similar to each other. The $N_{5}$ spectra exhibit asymmetric line shape while the $N_{4}$ spectra exhibit symmetric line shape. Furthermore, the integrated intensities of the $N_{5}$ and $N_{4}$ spectra have the same sign. However, the integrated intensities of the $N_{4}$ spectra are somewhat larger than those of the $N_{5}$ spectra. The ratio of the integrated intensities of the $N_{5}$ spectra to those of the $N_{4}$ spectra is $0.30,0.32$, and 0.34 for US, USe, and UTe, respectively, if we consider the total spectra. On the other hand, if we consider only the $\mathrm{U} 4 d \rightarrow 5 f$ spectra, the ratio of the $N_{5}$ spectra to those of the $N_{4}$ spectra is $0.35,0.39$, and 0.46 for US, USe, and UTe, respectively. It is found that the ratio increases from US to UTe. In contrast to the very good agreement between the calculated values of the XAS branching ratio of the $N_{4,5}$ and $M_{4,5}$ spectra, the calculated $N_{5}$ to $N_{4}$ ratio is notably larger than the calculated $M_{5}$ to $M_{4}$ ratio. This might mean that the XMCD spectra depend more strongly on the initial core states than the XAS spectra. The $N_{5}$ to $N_{4}$ ratio experimentally observed in US is 0.111 and that observed in USe is $0.116 .{ }^{31)}$ The discrepancy between the calculated and experimental values of the $N_{5}$ to $N_{4}$ ratio is notable. A possible origin is also the neglect of the spin-off-diagonal terms in the LSDA exchange potential as discussed in the previous subsection. The integrated intensity decreases from US to UTe. The ratio of the total integrated intensities of USe and UTe to that of US is 0.98 and 0.93 , respectively, if we consider the total spectra. On the other hand, if we consider only the U $4 d \rightarrow 5 f$ spectra, the ratio of USe and UTe to US is 0.99 and 0.96 , respectively. These results are completely the same as those found for the $M_{4,5}$ spectra.

In a theoretical work on $\mathrm{UFe}_{2},{ }^{24)}$ it was predicted that the XMCD intensity at the $N_{4,5}$ edges is one order of magnitude smaller than that at the $M_{4,5}$ edges. This prediction is in strong contradiction to our result that both the $N_{4,5}$ and $M_{4,5}$ peaks show the intensity of almost the same magnitude of about $10^{4} \mathrm{~cm}^{-1}$. A recent experimental study concluded that the intensity of the $N_{4,5}$ XMCD signal is comparable to that of the $M_{4,5}$ XMCD signal within experimental errors. ${ }^{31)}$ Although the investigated materials are different, it is hardly expected that the material difference is the origin of the discrepancy in the calculated XMCD intensity. It was also predicted that the $N_{5}$ to $N_{4}$ ratio of USe is larger by about 50-100\% than that of US. ${ }^{28)}$ This is also in contradiction to our result that the $N_{5}$ to $N_{4}$ ratio of USe is larger 
by at most about $10 \%$ than that of US. The experimental $N_{5}$ to $N_{4}$ ratio of USe is larger by only about $5 \%,{ }^{31)}$ in better agreement with our result. To resolve these contradictions, both theoretical and experimental studies are needed in the future.

\subsection{Sum-rule analysis}

We now study the orbital angular momentum $\left\langle L_{z}\right\rangle$ and the effective spin angular momentum $\left\langle S_{\mathrm{e}}\right\rangle$ of US, USe, and UTe using the XMCD sum rules. ${ }^{14-17)}$ The results of the sum-rule analysis are given in Tables I and II for $\left\langle L_{z}\right\rangle$ and $\left\langle S_{\mathrm{e}}\right\rangle$, respectively. The values of $\left\langle L_{z}\right\rangle$ and $\left\langle S_{\mathrm{e}}\right\rangle$ shown in the rows labeled "FFLCAO" are the results of the FFLCAO calculations. It is worth mentioning that we calculated $\left\langle T_{z}\right\rangle$ directly using the real space numerical integration adopted in the FFLCAO method; the calculated values are $0.21,0.23$, and 0.27 for US, USe, and UTe, respectively. Thus, the contribution of $\left\langle T_{z}\right\rangle$ to $\left\langle S_{\mathrm{e}}\right\rangle$ is found to be comparable to that of $\left\langle S_{z}\right\rangle$ as suggested in previous studies although our calculated values of $\left\langle T_{z}\right\rangle$ are smaller than the previous estimation of 0.3-0.4. ${ }^{22,29-31)}$ As already pointed out at the end of $\S 3.1$, the origin of this underestimation is most likely the neglect of the spin-off-diagonal terms in the LSDA exchange potential. ${ }^{22)}$ The large contribution of $\left\langle T_{z}\right\rangle$ to $\left\langle S_{\mathrm{e}}\right\rangle$ in $\mathrm{U} X$ is in strong contrast to its negligible contribution in $3 d$ transition metals. ${ }^{19,20)}$ We show $\rho$ and $\left\langle L_{z}\right\rangle$ in Table I as well as $\delta$ and $\left\langle S_{\mathrm{e}}\right\rangle$ in Table II. It is worth mentioning that $\rho$ and $\delta$ are determined directly from the XAS and XMCD spectra in contrast to $\left\langle L_{z}\right\rangle$ and $\left\langle S_{\mathrm{e}}\right\rangle$, which require the number of electrons in the $\mathrm{U} 5 f$ shell, $n_{5 f}$. This is one problem associated with the application of the XMCD sum rules because the concept of a shell no longer exists in solid state; it is necessary for obtaining $n_{5 f}$ to calculate it theoretically with a sufficiently reasonable procedure. For this purpose, the MPA is also useful. The values of $n_{5 f}$ obtained using the FFLCAO calculations with the MPA are 3.14, 3.17, and 3.20 for US, USe, and UTe, respectively. Our result is in agreement with the experimental and theoretical results that both US and USe have an electronic configuration close to $5 f^{3}$ with $n_{5 f}$ of USe being slightly larger than that of US. ${ }^{31,32)}$ In the tables, we show the values of $\left\langle L_{z}\right\rangle$ and $\left\langle S_{\mathrm{e}}\right\rangle$ calculated using $n_{5 f}=2.8,3.0$, and 3.2 so as to cover the range of interest and to understand the dependence of $\left\langle L_{z}\right\rangle$ and $\left\langle S_{\mathrm{e}}\right\rangle$ on $n_{5 f}$ in the vicinity of $n_{5 f}=3.0$.

In Table I, we find that the values of $\left\langle L_{z}\right\rangle$ obtained using the sum-rule analysis are in agreement with those obtained using the FFLCAO calculations within about 10 to $20 \%$ for the $\mathrm{U} M_{4,5}$ edges and about 5 to $15 \%$ for the $\mathrm{U} N_{4,5}$ edges if we consider only the $\mathrm{U} 3 d \rightarrow 5 f$ and $4 d \rightarrow 5 f$ spectra; it is found that the error increases from US to UTe for both the U $M_{4,5}$ and $N_{4,5}$ edges. On the contrary, the agreement is extremely imperfect if we consider the total spectra. This means that an appropriate extraction of the transitions relevant to the sum-rule analysis is indispensable for obtaining reliable values of $\left\langle L_{z}\right\rangle$ when employing the sum-rule analysis regardless of whether the analysis is experimental or theoretical. Furthermore, it is also worth mentioning that the agreement between the calculated and experimental values of 
$\rho$ is considerably good for the U $N_{4,5}$ edges of US and USe, as is also the case for $\left\langle L_{z}\right\rangle$. On the other hand, in Table II, we find that the values of $\left\langle S_{\mathrm{e}}\right\rangle$ obtained using the sum-rule analysis are in agreement with those obtained using the FFLCAO calculations within about -10 to $-20 \%$ for the $\mathrm{U} M_{4,5}$ edges and about -30 to $-35 \%$ for the $\mathrm{U} N_{4,5}$ edges. It is thus found that the error of the spin sum rule for the $\mathrm{U} N_{4,5}$ edges is considerably larger than the errors of the spin sum rule for the $\mathrm{U} M_{4,5}$ edges and the orbital sum rule for both the $\mathrm{U} M_{4,5}$ and $N_{4,5}$ edges.

The reason why the spin sum rule for $\mathrm{U} N_{4,5}$ edges does not work well can be understood as follows. The spin sum rule is derived assuming that the core spin-orbit splitting is sufficiently larger than the magnitudes of other interactions that mix the two partners of a spin-orbitsplit edge. ${ }^{15)}$ However, this condition is not always satisfied mainly due to the core-valence Coulomb interaction. ${ }^{21)}$ Actually, in our results, the error of the spin sum rule found for the $\mathrm{U} N_{4,5}$ edges is larger than almost $30 \%$ whereas the one found for the $\mathrm{U} M_{4,5}$ edges is less than $20 \%$. This may be due to the fact that the spin-orbit splitting of the $\mathrm{U} N_{4,5}$ edges, $41 \mathrm{eV}$, is much smaller than that of the $\mathrm{U} M_{4,5}$ edges, $180 \mathrm{eV}$; the former splitting is comparable to the magnitude of the core-valence Coulomb interaction whereas the latter splitting is considerably larger than the magnitude of this interaction. Thus, we may conclude that the larger error associated with the spin sum rule for the $\mathrm{U} N_{4,5}$ edges is due to the mixing of the two spin-orbit-split partners caused by the core-valence Coulomb interaction.

We have also pointed out in the above that the error of the orbital sum rule increases from US to UTe for both the U $M_{4,5}$ and $N_{4,5}$ edges. This might be understood as follows. Analyzing the partial densities of states of $U X$ calculated using the FFLCAO method, we found that the hybridization of the $n p$ atomic orbitals of the $X$ atom $(n=3,4$, and 5 for $X=\mathrm{S}$, Se, and Te, respectively), which is a possible origin of the error of the orbital sum rule, increases from US to UTe; out of $6 n p$ orbitals of $X, 1.8,2.0$, and 2.2 orbitals for $X=\mathrm{S}$, Se, and $\mathrm{Te}$, respectively, contribute to the conduction bands which mainly consist of the $\mathrm{U} 5 f$ atomic orbitals. Thus, it seems that the orbital sum rule works better for lighter $X$ atoms. On the contrary, the error of the spin sum rule does not depend on the degree of hybridization of the $X n p$ atomic orbitals probably because the spin degree of freedom is the internal one, not coupling directly to the external environment.

We next study the spin magnetic moment $M_{\text {spin }}$, the orbital magnetic moment $M_{\text {orb }}$, and the total magnetic moment $M_{\text {tot }}$ of US, USe, and UTe using the XMCD sum rules. The values of $M_{\text {spin }}$ with those of $\left\langle S_{z}\right\rangle$ are shown in Table III and the values of $M_{\text {orb }}$ with those of $M_{\text {tot }}=$ $M_{\text {spin }}+M_{\text {orb }}$ are shown in Table IV. To obtain $M_{\text {orb }}$, we use the relation $M_{\text {orb }}=-\left\langle L_{z}\right\rangle \mu_{\mathrm{B}}$ straightforwardly. On the other hand, to obtain $M_{\text {spin }}$, we use the relation $M_{\text {spin }}=-2\left\langle S_{z}\right\rangle \mu_{\mathrm{B}}$, which needs the values of $\left\langle T_{z}\right\rangle$ when using the spin sum rule. As already mentioned above, the values of $\left\langle T_{z}\right\rangle$ are calculated directly using the FFLCAO method. It should again be 
noted that, in agreement with previous results for US, the $\left\langle T_{z}\right\rangle$ term is very large and cannot be ignored even though the $\mathrm{U}$ atoms in $\mathrm{U} X$ are in a cubic environment. ${ }^{22,29-31)}$ Finally, we compare the values of $M_{\text {tot }}$ calculated using the FFLCAO method as well as those obtained using the sum-rule analysis with those obtained by the saturation magnetization measurement and the neutron diffraction experiments. ${ }^{41-43)}$ The calculated values of $M_{\text {tot }}$ are considerably smaller than experimental ones as usually attributed to the underestimation of the orbital magnetic moment in the LSDA calculations. ${ }^{27}$ ) This underestimation is likely the origin of the discrepancy between the experimental and calculated values of $M_{\text {tot }}$ although more detailed study is needed in the future.

\section{Conclusions}

We have studied the XMCD in $\mathrm{U} X$ at the $\mathrm{U} M_{4,5}$ and $N_{4,5}$ edges and examined the applicability of the XMCD sum rules to UX, using the FFLCAO method and employing the MPA as a procedure for extracting the transitions relevant to the sum-rule analysis. As a result, we have found that the orbital sum rule is valid to $10-20 \%$ for the $M_{4,5}$ edges and valid to $5-15 \%$ for the $N_{4,5}$ edges. On the other hand, we have found that the spin sum rule is valid to $10-20 \%$ for the $M_{4,5}$ edges whereas valid to $30-35 \%$ for the $N_{4,5}$ edges. Furthermore, we have found that our results are consistent with the recent experimental observation that the intensity of the $N_{4,5} \mathrm{XMCD}$ signal is comparable to that of the $M_{4,5} \mathrm{XMCD}$ signal, although contradicting the previous theoretical prediction that the XMCD intensity at the $N_{4,5}$ edges is one order of magnitude smaller than that at the $M_{4,5}$ edges. 
Table I. Values of $\rho$ and $\left\langle L_{z}\right\rangle$ calculated using sum-rule analysis of XAS and XMCD spectra at U $M_{4,5}$ and $N_{4,5}$ edges of US, USe, and UTe. The values of $\left\langle L_{z}\right\rangle$ calculated using the FFLCAO method are also shown. In calculating $\left\langle L_{z}\right\rangle$ with the orbital sum rule, $n_{5 f}=2.8,3.0$, and 3.2 are used so as to cover the range of interest. Shown in parentheses are the errors with respect to the corresponding FFLCAO results.

\begin{tabular}{|c|c|c|c|c|c|}
\hline & & $\rho$ & & $\left\langle L_{z}\right\rangle$ & \\
\hline \multirow[t]{9}{*}{ US } & \multirow[t]{2}{*}{ FFLCAO } & & \multicolumn{3}{|c|}{-2.40} \\
\hline & & & $n_{5 f}=2.8$ & $n_{5 f}=3.0$ & $n_{5 f}=3.2$ \\
\hline & $M_{4,5}:$ Total & -0.035 & $-1.19(-50 \%)$ & $-1.16(-52 \%)$ & $-1.14(-53 \%)$ \\
\hline & $M_{4,5}: 3 d \rightarrow 5 f$ & -0.079 & $-2.65(+10 \%)$ & $-2.61(+9 \%)$ & $-2.56(+7 \%)$ \\
\hline & $N_{4,5}:$ Total & -0.028 & $-0.94(-61 \%)$ & $-0.92(-62 \%)$ & $-0.91(-62 \%)$ \\
\hline & $N_{4,5}: 4 d \rightarrow 5 f$ & -0.074 & $-2.50(+4 \%)$ & $-2.45(+2 \%)$ & $-2.41(\quad 0 \%)$ \\
\hline & $M_{4,5}:$ Expt. ${ }^{\text {a) }}$ & -0.100 & -3.36 & -3.30 & -3.24 \\
\hline & $M_{4,5}:$ Expt. ${ }^{\mathrm{b})}$ & -0.079 & -2.65 & -2.61 & -2.56 \\
\hline & $N_{4,5}:$ Expt. ${ }^{\mathrm{c})}$ & -0.073 & -2.45 & -2.41 & -2.37 \\
\hline \multirow[t]{7}{*}{ USe } & \multirow[t]{2}{*}{ FFLCAO } & & \multicolumn{3}{|c|}{-2.71} \\
\hline & & & $n_{5 f}=2.8$ & $n_{5 f}=3.0$ & $n_{5 f}=3.2$ \\
\hline & $M_{4,5}:$ Total & -0.040 & $-1.36(-50 \%)$ & $-1.33(-51 \%)$ & $-1.31(-52 \%)$ \\
\hline & $M_{4,5}: 3 d \rightarrow 5 f$ & -0.091 & $-3.07(+13 \%)$ & $-3.01(+11 \%)$ & $-2.95(+9 \%)$ \\
\hline & $N_{4,5}:$ Total & -0.032 & $-1.08(-60 \%)$ & $-1.06(-61 \%)$ & $-1.04(-62 \%)$ \\
\hline & $N_{4,5}: 4 d \rightarrow 5 f$ & -0.086 & $-2.89(+7 \%)$ & $-2.84(+5 \%)$ & $-2.79(+3 \%)$ \\
\hline & $N_{4,5}:$ Expt. ${ }^{\mathrm{c})}$ & -0.082 & -2.76 & -2.71 & -2.66 \\
\hline \multirow[t]{6}{*}{ UTe } & \multirow[t]{2}{*}{ FFLCAO } & & \multicolumn{3}{|c|}{-3.18} \\
\hline & & & $n_{5 f}=2.8$ & $n_{5 f}=3.0$ & $n_{5 f}=3.2$ \\
\hline & $M_{4,5}:$ Total & -0.049 & $-1.65(-48 \%)$ & $-1.62(-49 \%)$ & $-1.59(-50 \%)$ \\
\hline & $M_{4,5}: 3 d \rightarrow 5 f$ & -0.114 & $-3.83(+20 \%)$ & $-3.76(+18 \%)$ & $-3.69(+16 \%)$ \\
\hline & $N_{4,5}:$ Total & -0.030 & $-1.01(-68 \%)$ & $-1.00(-69 \%)$ & $-0.98(-69 \%)$ \\
\hline & $N_{4,5}: 4 d \rightarrow 5 f$ & -0.107 & $-3.61(+14 \%)$ & $-3.55(+12 \%)$ & $-3.48(+9 \%)$ \\
\hline
\end{tabular}

a) Ref. 29

b) Ref. 30

c) Ref. 31 
Table II. Values of $\delta$ and $\left\langle S_{\mathrm{e}}\right\rangle$ calculated using sum-rule analysis of XAS and XMCD spectra at U $M_{4,5}$ and $N_{4,5}$ edges of US, USe, and UTe. The values of $\left\langle S_{\mathrm{e}}\right\rangle$ calculated using the FFLCAO method are also shown; the calculated $\left\langle T_{z}\right\rangle$ are $0.21,0.23$, and 0.27 for US, USe, and UTe, respectively. In calculating $\left\langle S_{\mathrm{e}}\right\rangle$ with the spin sum rule, $n_{5 f}=2.8,3.0$, and 3.2 are used so as to cover the range of interest. Shown in parentheses are the errors with respect to the corresponding FFLCAO results.

\begin{tabular}{|c|c|c|c|c|c|}
\hline \multirow{3}{*}{ US } & \multirow{3}{*}{ FFLCAO } & \multirow[t]{3}{*}{$\delta$} & \multicolumn{3}{|c|}{$\left\langle S_{\mathrm{e}}\right\rangle=\left\langle S_{z}\right\rangle+3\left\langle T_{z}\right\rangle$} \\
\hline & & & \multicolumn{3}{|c|}{$1.54=0.91+3 \times 0.21$} \\
\hline & & & $n_{5 f}=2.8$ & $n_{5 f}=3.0$ & $n_{5 f}=3.2$ \\
\hline & $M_{4,5}:$ Total & 0.039 & $0.66(-57 \%)$ & $0.64(-58 \%)$ & $0.63(-59 \%)$ \\
\hline & $M_{4,5}: 3 d \rightarrow 5 f$ & 0.078 & $1.30(-16 \%)$ & $1.28(-17 \%)$ & $1.26(-18 \%)$ \\
\hline & $N_{4,5}:$ Total & 0.026 & $0.44(-71 \%)$ & $0.43(-72 \%)$ & $0.42(-73 \%)$ \\
\hline & $N_{4,5}: 4 d \rightarrow 5 f$ & 0.063 & $1.06(-31 \%)$ & $1.04(-32 \%)$ & $1.02(-34 \%)$ \\
\hline & $M_{4,5}:$ Expt. ${ }^{\text {a) }}$ & 0.121 & 2.03 & 2.00 & 1.96 \\
\hline & $M_{4,5}:$ Expt. $^{\mathrm{b})}$ & 0.101 & 1.70 & 1.67 & 1.64 \\
\hline & $N_{4,5}:$ Expt. ${ }^{\text {c) }}$ & 0.092 & 1.55 & 1.52 & 1.49 \\
\hline \multirow[t]{7}{*}{ USe } & \multirow{2}{*}{\multicolumn{2}{|c|}{ FFLCAO }} & \multicolumn{3}{|c|}{$1.64=0.95+3 \times 0.23$} \\
\hline & & & $n_{5 f}=2.8$ & $n_{5 f}=3.0$ & $n_{5 f}=3.2$ \\
\hline & $M_{4,5}:$ Total & 0.043 & $0.73(-55 \%)$ & $0.72(-56 \%)$ & $0.70(-57 \%)$ \\
\hline & $M_{4,5}: 3 d \rightarrow 5 f$ & 0.085 & $1.43(-13 \%)$ & $1.40(-15 \%)$ & $1.34(-18 \%)$ \\
\hline & $N_{4,5}:$ Total & 0.029 & $0.48(-71 \%)$ & $0.47(-71 \%)$ & $0.47(-71 \%)$ \\
\hline & $N_{4,5}: 4 d \rightarrow 5 f$ & 0.069 & $1.16(-29 \%)$ & $1.14(-30 \%)$ & $1.12(-32 \%)$ \\
\hline & $N_{4,5}:$ Expt. ${ }^{\mathrm{c})}$ & 0.102 & 1.71 & 1.68 & 1.65 \\
\hline \multirow[t]{6}{*}{ UTe } & FFLCAO & & \multicolumn{3}{|c|}{$1.85=1.04+3 \times 0.27$} \\
\hline & & & $n_{5 f}=2.8$ & $n_{5 f}=3.0$ & $n_{5 f}=3.2$ \\
\hline & $M_{4,5}:$ Total & 0.051 & $0.86(-54 \%)$ & $0.85(-54 \%)$ & $0.83(-55 \%)$ \\
\hline & $M_{4,5}: 3 d \rightarrow 5 f$ & 0.095 & $1.59(-14 \%)$ & $1.57(-15 \%)$ & $1.54(-17 \%)$ \\
\hline & $N_{4,5}:$ Total & 0.026 & $0.44(-76 \%)$ & $0.43(-78 \%)$ & $0.42(-77 \%)$ \\
\hline & $N_{4,5}: 4 d \rightarrow 5 f$ & 0.077 & $1.29(-30 \%)$ & $1.26(-32 \%)$ & $1.24(-33 \%)$ \\
\hline
\end{tabular}

a) Ref. 29

b) Ref. 30

c) Ref. 31 
Table III. Values of $\left\langle S_{z}\right\rangle$ and $M_{\text {spin }}$ (in unit of $\mu_{\mathrm{B}}$ ) calculated using sum-rule analysis of XAS and XMCD spectra at $\mathrm{U} M_{4,5}$ and $N_{4,5}$ edges of US, USe, and UTe, adopting $\left\langle T_{z}\right\rangle$ calculated using the FFLCAO method. The values of $\left\langle S_{z}\right\rangle$ and $M_{\text {spin }}$ calculated using the FFLCAO method are also shown. In calculating $\left\langle S_{z}\right\rangle$ and $M_{\text {spin }}$ with the spin sum rule, $n_{5 f}=2.8,3.0$, and 3.2 are used so as to cover the range of interest.

\begin{tabular}{|c|c|c|c|c|c|c|c|c|}
\hline \multirow{3}{*}{ US } & \multirow{3}{*}{ FFLCAO } & \multirow{3}{*}{$\begin{array}{l}\left\langle T_{z}\right\rangle \\
0.21\end{array}$} & \multicolumn{3}{|c|}{$\left\langle S_{z}\right\rangle$} & \multicolumn{3}{|c|}{$M_{\text {spin }}$} \\
\hline & & & \multicolumn{3}{|c|}{0.91} & \multicolumn{3}{|c|}{-1.82} \\
\hline & & & $n_{5 f}=2.8$ & $n_{5 f}=3.0$ & $n_{5 f}=3.2$ & $n_{5 f}=2.8$ & $n_{5 f}=3.0$ & $n_{5 f}=3.2$ \\
\hline & $M_{4,5}:$ Total & & 0.03 & 0.01 & 0.00 & -0.06 & -0.02 & 0.00 \\
\hline & $M_{4,5}: 3 d \rightarrow 5 f$ & & 0.67 & 0.65 & 0.63 & -1.34 & -1.30 & -1.26 \\
\hline & $N_{4,5}:$ Total & & -0.19 & -0.20 & -0.19 & 0.38 & 0.40 & 0.38 \\
\hline & $N_{4,5}: 4 d \rightarrow 5 f$ & & 0.43 & 0.41 & 0.39 & -0.86 & -0.82 & -0.78 \\
\hline \multirow[t]{6}{*}{ USe } & FFLCAO & 0.23 & \multicolumn{3}{|c|}{0.95} & \multicolumn{3}{|c|}{-1.90} \\
\hline & & & $n_{5 f}=2.8$ & $n_{5 f}=3.0$ & $n_{5 f}=3.2$ & $n_{5 f}=2.8$ & $n_{5 f}=3.0$ & $n_{5 f}=3.2$ \\
\hline & $M_{4,5}:$ Total & & 0.04 & 0.03 & 0.01 & -0.08 & -0.06 & -0.02 \\
\hline & $M_{4,5}: 3 d \rightarrow 5 f$ & & 0.74 & 0.71 & 0.65 & -1.48 & -1.42 & -1.30 \\
\hline & $N_{4,5}:$ Total & & -0.21 & -0.22 & -0.22 & 0.42 & 0.44 & 0.44 \\
\hline & $N_{4,5}: 4 d \rightarrow 5 f$ & & 0.47 & 0.45 & 0.43 & -0.94 & -0.90 & -0.86 \\
\hline \multirow[t]{6}{*}{ UTe } & FFLCAO & 0.27 & \multicolumn{3}{|c|}{1.04} & \multicolumn{3}{|c|}{-2.08} \\
\hline & & & $n_{5 f}=2.8$ & $n_{5 f}=3.0$ & $n_{5 f}=3.2$ & $n_{5 f}=2.8$ & $n_{5 f}=3.0$ & $n_{5 f}=3.2$ \\
\hline & $M_{4,5}:$ Total & & 0.05 & 0.04 & 0.02 & -0.10 & -0.08 & -0.04 \\
\hline & $M_{4,5}: 3 d \rightarrow 5 f$ & & 0.78 & 0.76 & 0.73 & -1.56 & -1.52 & -1.46 \\
\hline & $N_{4,5}:$ Total & & -0.37 & -0.38 & -0.39 & 0.74 & 0.76 & 0.78 \\
\hline & $N_{4,5}: 4 d \rightarrow 5 f$ & & 0.48 & 0.45 & 0.43 & -0.96 & -0.90 & -0.86 \\
\hline
\end{tabular}


Table IV. Values of $M_{\text {orb }}$ (in unit in $\mu_{\mathrm{B}}$ ) and $M_{\text {tot }}$ (in unit of $\mu_{\mathrm{B}}$ ) calculated using sum-rule analysis of XAS and XMCD spectra at U $M_{4,5}$ and $N_{4,5}$ edges of US, USe, and UTe. In calculating the contribution of $M_{\text {spin }}$ to $M_{\text {tot }}=M_{\text {spin }}+M_{\text {orb }}$, the values of $\left\langle T_{z}\right\rangle$ calculated using the FFLCAO method are adopted. The values of $M_{\text {orb }}$ and $M_{\text {tot }}$ calculated using the FFLCAO method are also shown. In calculating $M_{\text {orb }}$ and $M_{\text {tot }}$ with the orbital and spin sum rules, $n_{5 f}=2.8,3.0$, and 3.2 are used so as to cover the range of interest.

\begin{tabular}{|c|c|c|c|c|c|c|c|}
\hline \multirow{3}{*}{ US } & \multirow{3}{*}{ FFLCAO } & \multicolumn{3}{|c|}{$M_{\text {orb }}$} & \multicolumn{3}{|c|}{$M_{\text {tot }}$} \\
\hline & & \multicolumn{3}{|c|}{2.40} & \multicolumn{3}{|c|}{0.58} \\
\hline & & $n_{5 f}=2.8$ & $n_{5 f}=3.0$ & $n_{5 f}=3.2$ & $n_{5 f}=2.8$ & $n_{5 f}=3.0$ & $n_{5 f}=3.2$ \\
\hline & $M_{4,5}:$ Total & 1.19 & 1.16 & 1.14 & 1.13 & 1.14 & 1.14 \\
\hline & $M_{4,5}: 3 d \rightarrow 5 f$ & 2.65 & 2.61 & 2.56 & 1.31 & 1.31 & 1.30 \\
\hline & $N_{4,5}:$ Total & 0.94 & 0.92 & 0.91 & 1.32 & 1.32 & 1.29 \\
\hline & $N_{4,5}: 4 d \rightarrow 5 f$ & 2.50 & 2.45 & 2.41 & 1.64 & 1.63 & 1.63 \\
\hline & Expt. $^{\text {a) }}$ & & & & & 1.55 & \\
\hline & Expt. ${ }^{b)}$ & & & & & 1.70 & \\
\hline \multirow[t]{8}{*}{ USe } & \multirow[t]{2}{*}{ FFLCAO } & \multicolumn{3}{|c|}{2.71} & \multicolumn{3}{|c|}{0.81} \\
\hline & & $n_{5 f}=2.8$ & $n_{5 f}=3.0$ & $n_{5 f}=3.2$ & $n_{5 f}=2.8$ & $n_{5 f}=3.0$ & $n_{5 f}=3.2$ \\
\hline & $M_{4,5}:$ Total & 1.36 & 1.33 & 1.31 & 1.28 & 1.27 & 1.29 \\
\hline & $M_{4,5}: 3 d \rightarrow 5 f$ & 3.07 & 3.01 & 2.95 & 1.59 & 1.59 & 1.65 \\
\hline & $N_{4,5}:$ Total & 1.08 & 1.06 & 1.04 & 1.50 & 1.50 & 1.48 \\
\hline & $N_{4,5}: 4 d \rightarrow 5 f$ & 2.89 & 2.84 & 2.79 & 1.95 & 1.94 & 1.93 \\
\hline & Expt. ${ }^{a)}$ & & & & & 1.81 & \\
\hline & Expt. ${ }^{c}$ & & & & & 2.0 & \\
\hline \multirow[t]{8}{*}{ UTe } & \multirow[t]{2}{*}{ FFLCAO } & \multicolumn{3}{|c|}{3.18} & \multicolumn{3}{|c|}{1.10} \\
\hline & & $n_{5 f}=2.8$ & $n_{5 f}=3.0$ & $n_{5 f}=3.2$ & $n_{5 f}=2.8$ & $n_{5 f}=3.0$ & $n_{5 f}=3.2$ \\
\hline & $M_{4,5}:$ Total & 1.65 & 1.62 & 1.59 & 1.55 & 1.54 & 1.55 \\
\hline & $M_{4,5}: 3 d \rightarrow 5 f$ & 3.83 & 3.76 & 3.69 & 2.27 & 2.24 & 2.23 \\
\hline & $N_{4,5}:$ Total & 1.01 & 1.00 & 0.98 & 1.75 & 1.76 & 1.76 \\
\hline & $N_{4,5}: 4 d \rightarrow 5 f$ & 3.61 & 3.55 & 3.48 & 2.65 & 2.65 & 2.62 \\
\hline & Expt. ${ }^{a)}$ & & & & & 1.91 & \\
\hline & Expt. ${ }^{c)}$ & & & & & 2.25 & \\
\hline
\end{tabular}

a) Ref. 41; bulk magnetic moment at saturation.

b) Ref. 42; neutron diffraction.

c) Ref. 43; neutron diffraction. 

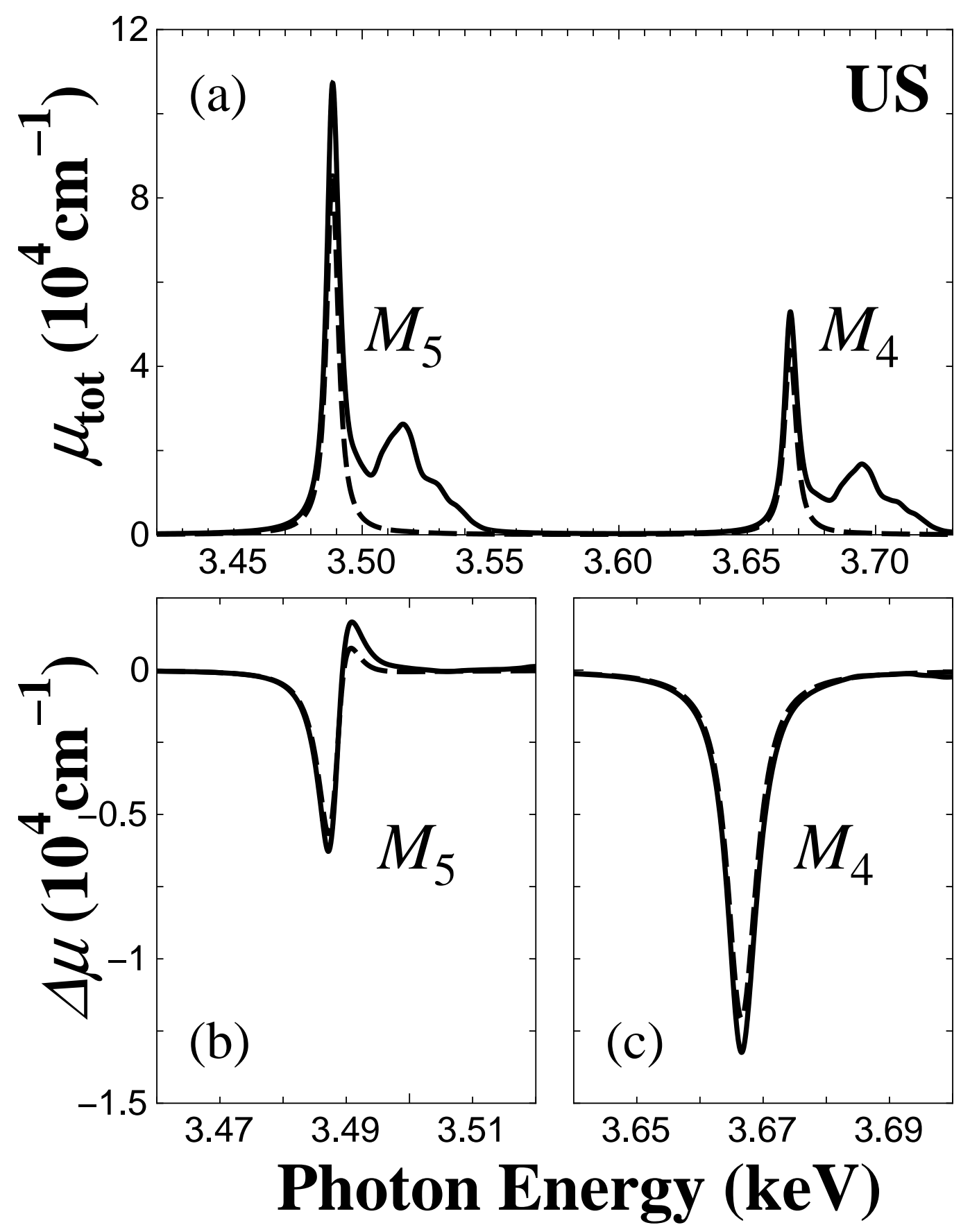

Fig. 1. XAS and XMCD spectra at U $M_{4,5}$ edges of US calculated using FFLCAO method: (a) XAS spectra, (b) XMCD spectra for $M_{5}$ edge, and (c) XMCD spectra for $M_{4}$ edge. Solid lines represent the total spectra involving all transitions while dashed lines the spectra originated in the $\mathrm{U} 3 d \rightarrow$ $5 f$ transitions extracted using the MPA. 

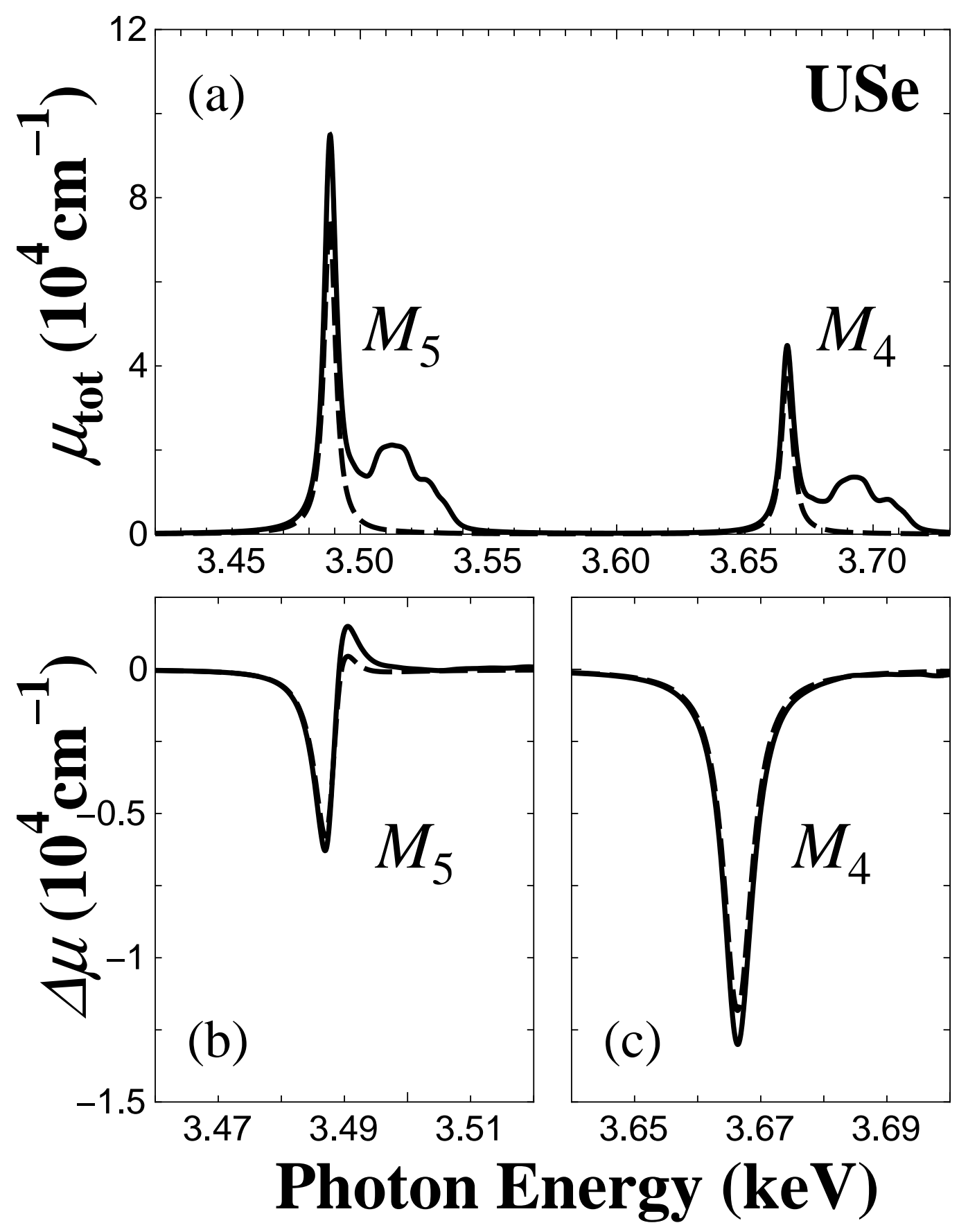

Fig. 2. XAS and XMCD spectra at $\mathrm{U} M_{4,5}$ edges of USe calculated using FFLCAO method: (a) XAS spectra, (b) XMCD spectra for $M_{5}$ edge, and (c) XMCD spectra for $M_{4}$ edge. Solid lines represent the total spectra involving all transitions while dashed lines the spectra originated in the $\mathrm{U} 3 d \rightarrow$ $5 f$ transitions extracted using the MPA. 

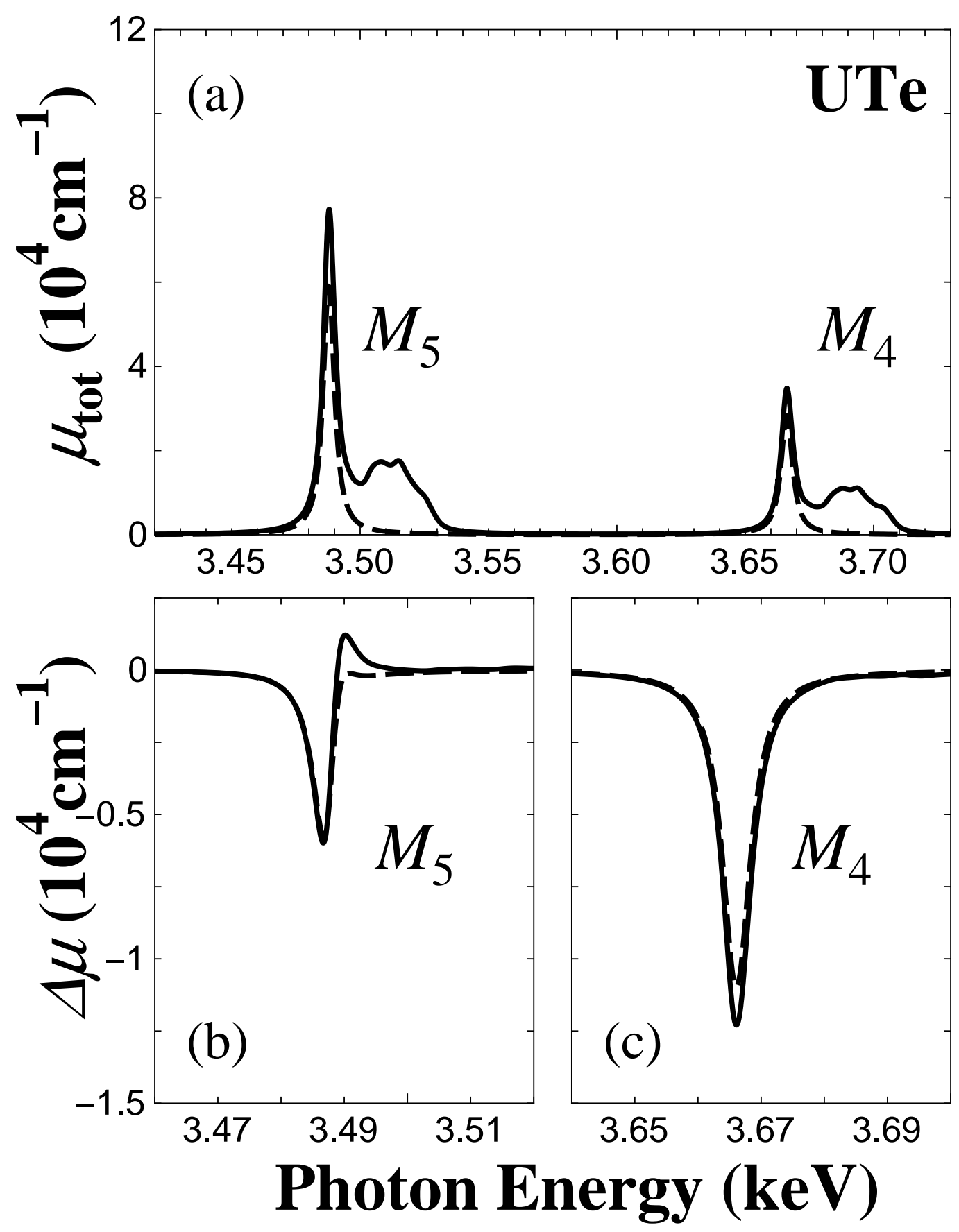

Fig. 3. XAS and XMCD spectra at $U M_{4,5}$ edges of UTe calculated using FFLCAO method: (a) XAS spectra, (b) XMCD spectra for $M_{5}$ edge, and (c) XMCD spectra for $M_{4}$ edge. Solid lines represent the total spectra involving all transitions while dashed lines the spectra originated in the $\mathrm{U} 3 d \rightarrow$ $5 f$ transitions extracted using the MPA. 

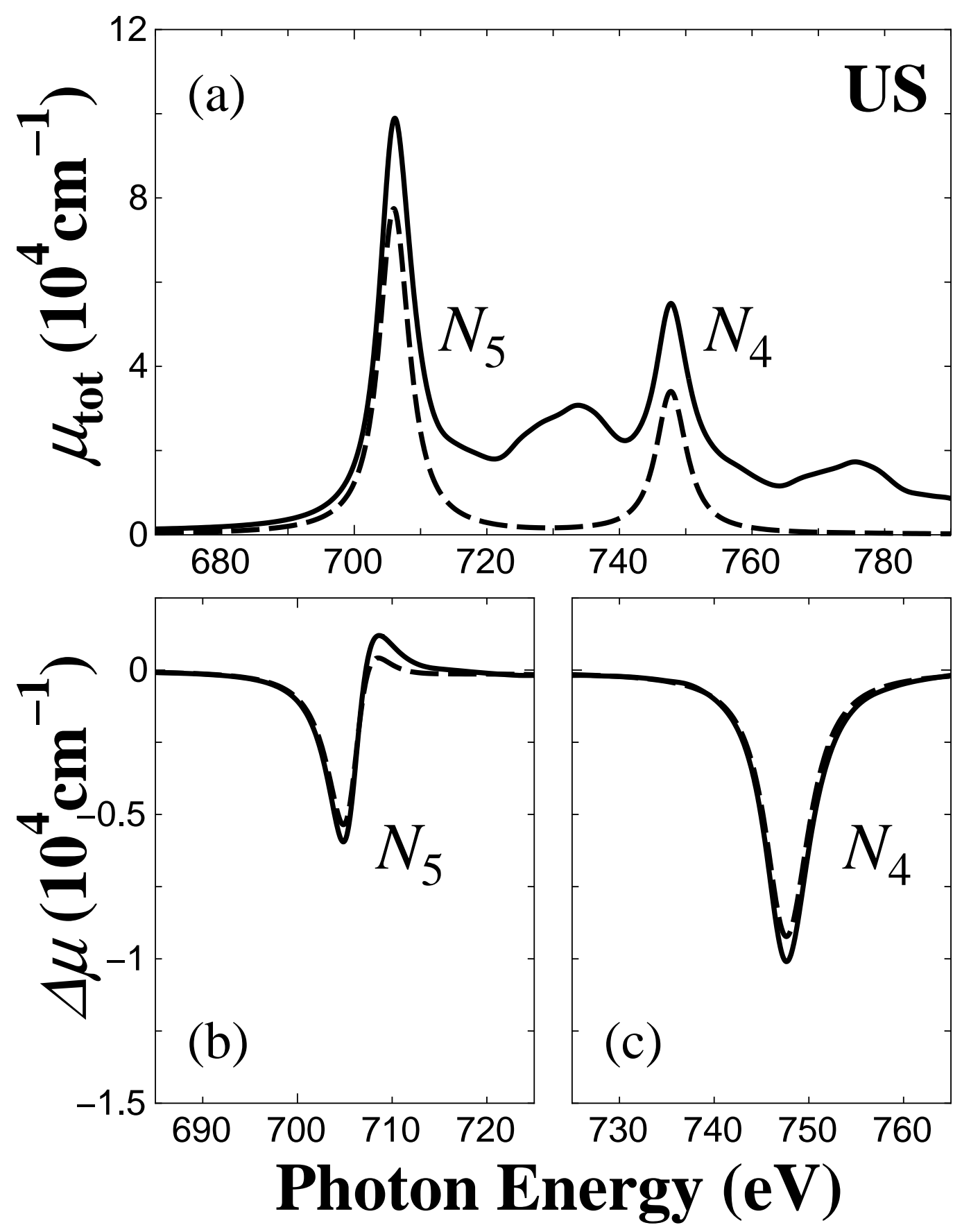

Fig. 4. XAS and XMCD spectra at U $N_{4,5}$ edges of US calculated using FFLCAO method: (a) XAS spectra, (b) XMCD spectra for $N_{5}$ edge, and (c) XMCD spectra for $N_{4}$ edge. Solid lines represent the total spectra involving all transitions while dashed lines the spectra originated in the $\mathrm{U} 4 d \rightarrow$ $5 f$ transitions extracted using the MPA. 

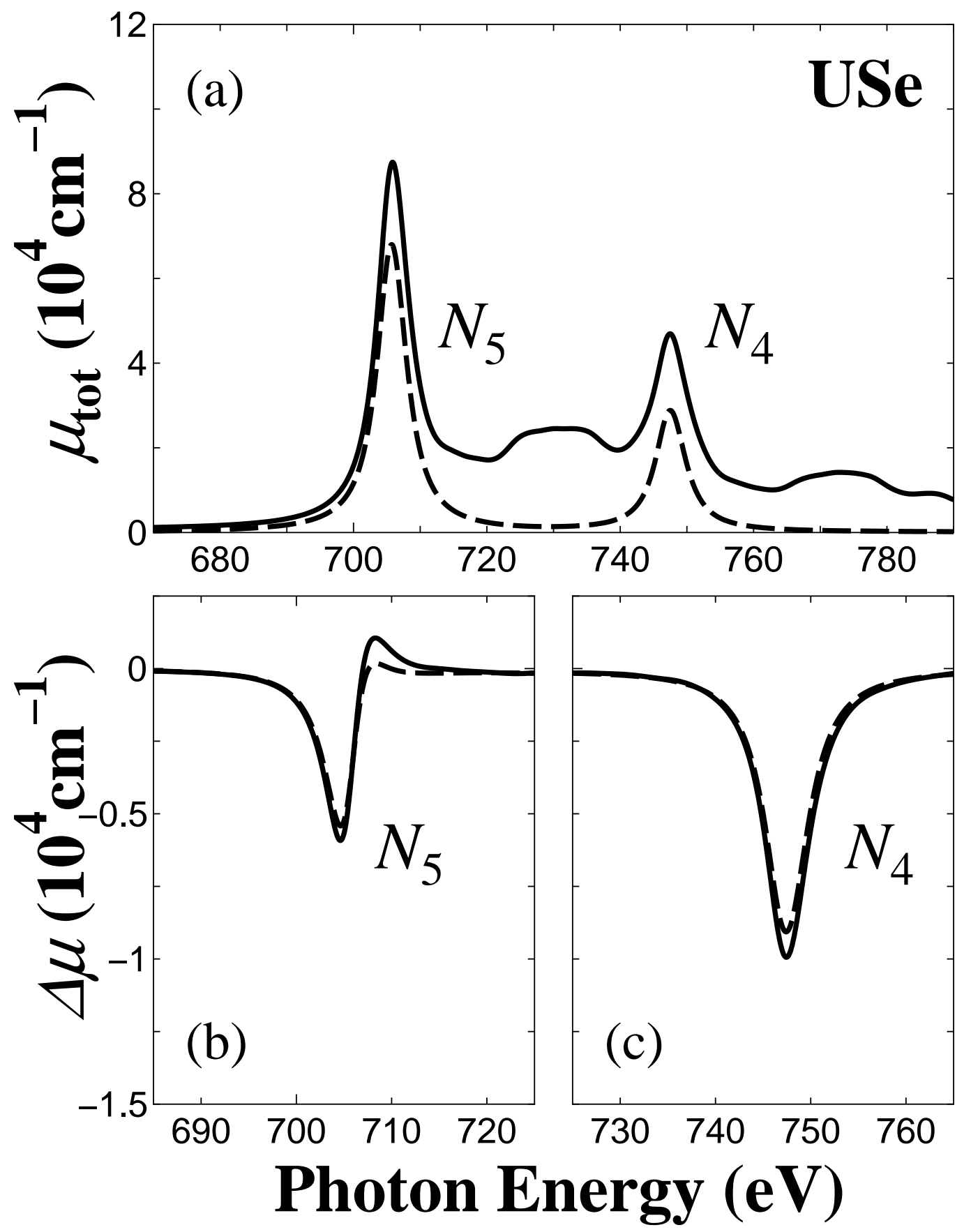

Fig. 5. XAS and XMCD spectra at U $N_{4,5}$ edges of USe calculated using FFLCAO method: (a) XAS spectra, (b) XMCD spectra for $N_{5}$ edge, and (c) XMCD spectra for $N_{4}$ edge. Solid lines represent the total spectra involving all transitions while dashed lines the spectra originated in the $\mathrm{U} 4 d \rightarrow$ $5 f$ transitions extracted using the MPA. 

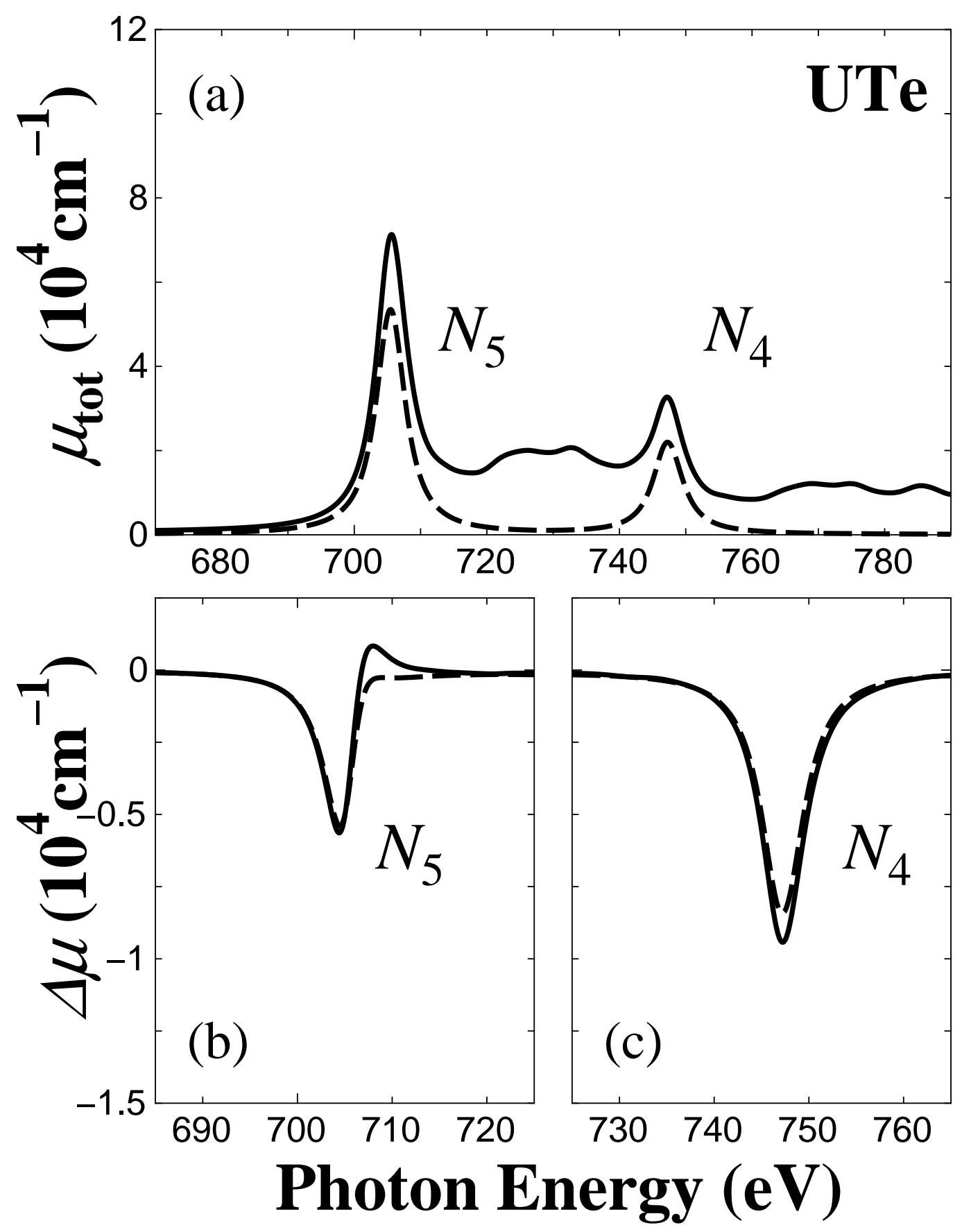

Fig. 6. XAS and XMCD spectra at U $N_{4,5}$ edges of UTe calculated using FFLCAO method: (a) XAS spectra, (b) XMCD spectra for $N_{5}$ edge, and (c) XMCD spectra for $N_{4}$ edge. Solid lines represent the total spectra involving all transitions while dashed lines the spectra originated in the $\mathrm{U} 4 d \rightarrow$ $5 f$ transitions extracted using the MPA. 


\section{References}

1) G. Schütz, W. Wagner, W. Wilhelm, P. Kienle, R. Zeller, R. Frahm, and G. Materlik: Phys. Rev. Lett. 58 (1987) 737.

2) H. Ebert and R. Zeller: Phys. Rev. B 42 (1990) 2744.

3) C. T. Chen, F. Sette, Y. Ma, and S. Modesti: Phys. Rev. B 42 (1990) 7262.

4) C. T. Chen, N. V. Smith, and F. Sette: Phys. Rev. B 43 (1991) 6785.

5) T. Koide, T. Shidara, M. Yuri, N. Kandaka, and H. Fukutani: Appl. Phys. Lett. 58 (1991) 2592.

6) T. Koide, T. Shidara, H. Fukutani, K. Yamaguchi, A. Fujimori, and S. Kimura: Phys. Rev. B 44 (1991) 4697.

7) A. Yoshida and T. Jo: J. Phys. Soc. Jpn. 60 (1991) 2098.

8) C. T. Chen, Y. U. Idzerda, H.-J. Lin, N. V. Smith, G. Meigs, E. Chaban, G. H. Ho, E. Pellegrin, and F. Sett: Phys. Rev. Lett. 75 (1995) 152.

9) J. Stöhr and H. König: Phys. Rev. Lett. 75 (1995) 3748.

10) E. Arola, P. Strange, and B. L. Gyorffy: Phys. Rev. B 55 (1997) 472.

11) A. Yaouanc, P. Dalmas de Réotier, G. van der Laan, A. Hiess, J. Goulon, C. Neumann, P. Lejay, and N. Sato: Phys. Rev. B 58 (1998) 8793.

12) P. Dalmas de Réotier, A. Yaouanc, G. van der Laan, N. Kernavanois, J.-P. Sanchez, J. L. Smith, A. Hiess, A. Huxley, and R. Rogalev: Phys. Rev. B 60 (1999) 10606.

13) R. Wu and A. J. Freeman: J. Magn. Magn. Mater. 200 (1999) 498.

14) B. T. Thole, P. Carra, F. Sette, and G. van der Laan: Phys. Rev. Lett. 68 (1992) 1943.

15) P. Carra, B. T. Thole, M. Altarelli, and X. Wang: Phys. Rev. Lett. 70 (1993) 694.

16) A. Ankudinov and J. J. Rehr: Phys. Rev. B 51 (1995) 1282.

17) G. van der Laan and B. T. Thole: Phys. Rev. B 53 (1996) 14458.

18) R. Wu, D. Wang, and A. J. Freeman: Phys. Rev. Lett. 71 (1993) 3581.

19) R. Wu and A. J. Freeman: Phys. Rev. Lett. 73 (1994) 1994.

20) R. Wu, D. Wang, and A. J. Freeman: J. Appl. Phys. 75 (1994) 5802.

21) Y. Teramura, A. Tanaka, and T. Jo: J. Phys. Soc. Jpn. 65 (1996) 1053.

22) T. Shishidou, T. Oguchi, and T. Jo: Phys. Rev. B 59 (1999) 6813.

23) T. Jo: J. Synchrotron Rad. 8 (2001) 115.

24) V. N. Antonov, B. N. Harmon, and A. N. Yaresko: Phys. Rev. B 68 (2003) 214424.

25) V. N. Antonov, B. N. Harmon, O. V. Andryushchenko, L. V. Bekenev, and A. N. Yaresko: Phys. Rev. B 68 (2003) 214425.

26) V. N. Antonov, B. N. Harmon, O. V. Andryushchenko, L. V. Bekenev, and A. N. Yaresko: Phys. Rev. B 68 (2003) 214426.

27) V. N. Antonov, B. N. Harmon, O. V. Andryushchenko, L. V. Bekenev, and A. N. Yaresko: Low Temp. Phys. 30 (2004) 305.

28) Electronic Structure and Magneto-Optical Properties of Solids, V. N. Antonov, B. N. Harmon, and A. N. Yaresko (Kluwer Academic, Dordrecht, 2004). Chap. 4.3.

29) S. P. Collins, D. Laundy, C. C. Tang, and G. van der Laan: J. Phys.: Condens. Matter 7 (1995) 9325 .

30) N. Kernavanois, P. Dalmas de Réotier, A. Yaouanc, J.-P. Sanchez, V. Honkimäki, T. Tschentscher, J. McCarthy, and O. Vogt: J. Phys.: Condens. Matter 13 (2001) 9677. 
31) T. Okane, Y. Takeda, J. Okamoto, K. Mamiya, T. Ohkochi, S. Fujimori, Y. Saitoh, H. Yamagami, A. Fujimori, A. Ochiai, and A. Tanaka: J. Phys. Soc. Jpn. 77 (2008) 024706.

32) H. Yamagami: J. Phys. Soc. Jpn. 67 (1998) 3176.

33) R. S. Mulliken: J. Chem. Phys. 23 (1955) 1833.

34) S. Suzuki and K. Nakao: J. Phys. Soc. Jpn. 68 (1999) 1982.

35) M.-F. Li, T. Ariizumi, and S. Suzuki: J. Phys. Soc. Jpn. 76 (2007) 054702.

36) S. Suzuki, M.-F. Li, and T. Ariizumi: J. Phys. Soc. Jpn. 77 (2008) 074703.

37) Handbook on the Physics and Chemistry of the Actinides, ed. A. J. Freeman and G. H. Lander (North-Holland, Amsterdam, 1985).

38) J. P. Perdew and A. Zunger: Phys. Rev. B 23 (1981) 5048.

$39)$ D. M. Ceperley and B. J. Alder: Phys. Rev. Lett. 45566 (1980) 566.

40) L.-K. Hua and Y. Wang: Applications of Number Theory to Numerical Analysis (Springer-Verlag, Berlin, 1981).

41) J. M. Fournier and R. Tróc: in Handbook on the Physics and Chemistry of the Actinides, ed. A. J. Freeman and G. H. Lander (North-Holland, Amsterdam, 1985) Vol. 2, p. 239.

42) F. A. Wedgwood: J. Phys. C: Solid State Phys. 5 (1972) 2427.

$43)$ F. A. Wedgwood and M. Kuznietz: J. Phys. C: Solid State Phys. 5 (1972) 3012. 